Article original

\title{
Distribution et incidence de la mosaïque du concombre (CMV) dans des bananeraies industrielles au Sud-Est de la Côte d'Ivoire.
}

\author{
Aka R. AkA ${ }^{1}$, Nazaire K. KouAssi ${ }^{1 *}$, Thérèse A. Agnéroh ${ }^{2}$, Nicaise A. AmANCHO ${ }^{1}$ \& Abdourahamane SangarÉ ${ }^{1}$ \\ ${ }^{1}$ Laboratoire Central de Biotechnologies, CNRA, 01 BP 1740, Abidjan, Côte d'Ivoire. \\ ${ }^{2}$ Laboratoire de Phytopathologie et de Biologie Végétale, INPHB, Ecole Supérieure d'Agronomie, BP 1313, Yamoussoukro, RCI. \\ * Auteur pour les correspondances (E-mail : kouassinazaire@yahoo.fr) \\ Reçu le 26-06-2008, accepté le 19-06-2009.
}

\section{Résumé}

La distribution et l'incidence du virus de la mosaïque du concombre (CMV) infectant le bananier ont été évaluées dans les bananeraies au Sud-Est de la Côte d'Ivoire. Pour ce faire, des prospections ont été effectuées dans des plantations industrielles des secteurs de Niéky (Dabou); Banacomoé (Abengourou) et Grand-fleuve (Tiassalé). Des données de relevés phytosanitaires relatives au CMV ont été recueillies et des échantillons de feuilles symptomatiques et asymptomatiques de bananiers de la variété "Grande naine" ont été collectés. Des diagnostics sérologiques utilisant les tests DAS-ELISA (Double Antibody Sandwich Enzyme Linked Immunosorbent Assay), ont permis de confirmer la présence du CMV dans ces échantillons. Dans la pépinière du secteur Banacomoé, le CMV a été diagnostiqué dans environ $50 \%$ de lots de plantules issus de micropropagation (vitroplants). L'incidence de la maladie enregistrée sur la base des symptômes observés dans les trois secteurs indiquent un taux variant de $5 \%$ à $25 \%$ avec une prédominance $(66,25 \%$ ) des symptômes sévères (pourriture du cœur) sur les plants de 3 mois d'âges. Cependant, les plantations de plus de trois mois d'âge et les plants de la collection in vivo de pieds mères utilisés pour la micropropagation n'ont présenté que de rares plants infectés. La répartition du CMV dans les plantations de moins de 3 mois a révélé une prédominance des foyers d'infection de type marginal.

Mots clés : Cucumber Mosaic Virus, DAS-ELISA, incidence, distribution, Musa spp., Côte d'Ivoire

\begin{abstract}
Distribution and incidence of Cucumber mosaic virus disease (CMVD) in some industrial banana plantations in the South-East of Côte d'lvoire.

This survey has been carried out in order to study the incidence and distribution of the Cucumber Mosaic Virus (CMV) infecting banana in the South-East part of Côte d'Ivoire. For this purpose, an assessment was conducted in the zones of Niéky (Dabou); Banacomoé (Abengourou) and Grand-fleuve (Tiassalé), in order to gather phytosanitary data concerning the occurrence of the CMV in the nursery and fields. Banana leaf samples of the "Grande naine"variety showing CMV symptoms and some without symptom were collected. The presence of the virus has been confirmed by serological tests using DAS-ELISA (Double Antibody Sandwich Enzyme Linked Immunosorbent Assay). About $50 \%$ of the tissue culture derived banana batches composing the nursery in a plantation of Abengourou was found to be infected by CMV. Based on symptoms observed, the incidence of the disease in these production sectors visited, varied from $5 \%$ to $25 \%$ with a predominance $(66,25 \%)$ of severe symptoms (heart-rot, symptoms) on plants of less than 3 month of age. However, plants beyond three months showed less CMV infected plants and less severe symptoms. Similarly, only few plants were infected in the germplasm containing mother plants used for micropropagation. In the field, banana plants showing CMV symptoms were more frequently distributed at the edge of the fields.
\end{abstract}

Key words: Cucumber Mosaic Virus, DAS-ELISA, incidence, distribution, Musa spp., Côte d'Ivoire. 


\section{Introduction}

Avec une production annuelle d'environ 95 millions de tonnes, la banane (Musa spp.) constitue un aliment de base pour environ 400 millions de personnes et une culture de rente aux retombées économiques majeures pour les pays du Sud (Caruana, 2007). Les bananes desserts du sousgroupe des Cavendish (génome de type AAA) sont produites principalement en Amérique latine, aux Caraïbes, en Afrique subsaharienne et en Asie du Sud-Est. En 2006, la production de banane de la Côte d'Ivoire a chuté à 235,000 tonnes alors qu'elle était de 270,000 tonnes en 2005 (FAOSTAT, 2009). En 2003, la Côte d'Ivoire et le Cameroun étaient les premiers pays africains fournisseurs de bananes dessert sur le marché de l'Union Européenne. Les variétés commercialisées sont la 'Grande naine', 'Poyo' et 'Williams' du sousgroupe des Cavendish (Kouassi, 2001). L'ensemble de ces cultivars, est sensible aux agressions biotiques que sont les mycoses, les bactérioses et les viroses qui occasionnent d'importantes pertes de rendement (Abadie et al., 2004). Cinq principaux virus infectant le bananier sont décrits à travers le monde à savoir, Banana Bunchy Top Virus (BBTV), Banana Streak Virus (BSV), Cucumber Mosaic Virus (CMV), Banana Bract Mosaic Virus (BBrMV) et le Banana Mild Mosaic Virus (BanMMV) (Lockhart, 2002). Les méthodes de lutte contre ces virus diffèrent cependant à cause de leurs caractéristiques biologiques et épidémiologiques spécifiques. Les pertes de production occasionnées par les viroses sont parfois très importantes. A titre d'exemple, les pertes de récoltes dues au BBTV au Pakistan sont estimées entre 90 et $100 \%$. Pour le BSV, ces pertes ont été supérieures à $40 \%$ aux Philippines et elles ont varié de 40 à $60 \%$ au Rwanda (Abadie et al., 2004).

La chlorose infectieuse du bananier ou la mosaïque du bananier, causée par "Cucumber mosaic virus" (CMV) est une maladie insidieuse présente dans tous les pays producteurs de banane. Elle se caractérise par une mosaïque chlorotique sur les jeunes feuilles, avec déformation de la feuille et un nanisme de la plante. Le virus est transmis par des pucerons, principalement Aphis gossypii, Rhopalosiphum maïdis, Myzus persicae et Macrosiphum euphorbia. La gamme d'hôtes du virus est extrêmement large et comporte plusieurs espèces de solanacées et de cucurbitacées (Lockhart, 2002). Aux Philippines où la maladie est endémique, il n'a pas encore été observé de fortes incidences sauf dans le sud Cotabato, où de sérieux dommages ont été rapportés sur certains cultivars des sous-groupes Cavendish et Saba. A Taiwan, des enquêtes ont révélé que l'incidence du CMV varie entre $1,9 \%$ et $8 \%$ (Hwang \& Su, 1998).

En Côte d'Ivoire, le CMV a été signalé sur le bananier depuis plusieurs dizaines d'années (Lassoudière, 1974 ; Fauquet \& Thouvenel, 1982 ; Dieckman \& Putter, 1996) sans qu'elle ne présente pour le moment de grandes conséquences économiques (Jones, 1994). Cette virose pourrait cependant avoir des conséquences économiques importantes si des mesures préventives ne sont pas adoptées par les planteurs. L'utilisation et les échanges de matériel végétal entre producteurs peuvent constituer une importante source de dissémination de cette virose. En Côte d'Ivoire, seules certaines sociétés industrielles se préoccupent de cette virose en adoptant des mesures de lutte préventives telles que l'identification et l'élimination systématique des plants malades. Pourtant, au regard du mode de transmission du virus et de l'utilisation des rejets directement en plantations par certains planteurs ou pour la production de vitroplants par d'autres, des études sont nécessaires pour prévenir la dissémination de cette virose. Ainsi, l'évaluation de l'incidence, la répartition spatiale de la virose dans des bananeraies de la Côte d'Ivoire et particulièrement le diagnostic sérologique des pieds mère du germoplasme, sont autant de travaux préliminaires à mener. Cette étude a donc porté sur l'évaluation de l'incidence et la distribution de la virose du CMV dans les bananeraies industrielles de trois localités du Sud-Est de la Côte d'Ivoire. Pour ce faire, le diagnostic sérologique de plants apparemment sains, de plants suspects ou symptomatiques a été réalisé dans le but de confirmer ou d'identifier l'agent causal de la maladie.

\section{Matériel et méthodes}

\subsection{Matériel biologique}

Le matériel végétal est constitué de vitroplants de la variété de bananes 'Grande naine' au stade 
pépinière, 3 mois après planting ou stade 3 MAP et 8 MAP des secteurs de production de Niéky (Dabou, 980 ha environ) de Banacomoé (Abengourou, 670 ha environ) et de Grand-fleuve (Tiassalé, 1000 ha environ). Des plants apparemment sains de stades phénologiques différents issus du germoplasme de bananiers du secteur Banacomoé (Abengourou), riche d'environ 1800 génotypes ont servi de témoins de contrôle pour les tests sérologiques.

Le matériel viral est constitué de 52 isolats du CMV issus des plants de bananier présentant les symptômes caractéristiques du CMV récoltés dans des plantations industrielles au sud de la Côte d'Ivoire.

\subsection{Méthode de prospections et d'échantil- lonnage}

Les prospections des plantations de bananiers ont été effectuées dans les trois secteurs de productions de la banane dessert en Côte d'Ivoire que sont les secteurs Niéky/Dabou (situé au Sud à $46 \mathrm{~km}$ d'Abidjan) ; Banacomoé/ Abengourou (situé à l'Est à $180 \mathrm{~km}$, d'Abidjan) et Grand-fleuve/Tiassalé (situé au Sud, à 130 km d'Abidjan). Ces secteurs comportent des plantations subdivisées en blocs, eux-mêmes composés d'une dizaine de carrés (sous parcelles) dont les superficies varient entre 2 et 4 ha en moyenne. Le nombre de carrés visités a été fonction du nombre de parcelles et des stades végétatifs des bananiers. Ainsi, pour le secteur Niéky, 10 carrés dont les plants sont âgés de 3 MAP et 10 autres possédant des plants âgés de 3 à $8 \mathrm{MAP}$ ont été visités. Pour le secteur Grandfleuve, 2 carrés de plants âgés de 3 MAP et 10 carrés de plants âgés 3 à 8 MAP ont été visités. Pour le secteur Banacomoé, 5 carrés dont les plants sont âgés de 3 MAP et 8 carrés présentant des plants âgés de 3 à 8 MAP ont été visités. En outre, les données portant sur l'effectif des plants infectés par le CMV en pépinière et en plantation, disponibles chez les contrôleurs phytosanitaires, ont été exploitées. Au cours de ces visites, 52 échantillons de feuilles de bananiers suspects ou présentant des symptômes de CMV et 7 échantillons de feuilles apparemment saines, issus du germoplasme ont été collectés pour des tests de diagnostic. L'estimation du nombre de plants malades a été faite à partir des relevés phytosanitaires disponibles pour chaque plantation.

\subsection{Evaluation de la sévérité des symptômes sur les plants}

Le degré de sévérité des symptômes foliaires a été évalué sur les plants en plantation avec l'échelle de Cours (Cours, 1950 ; Fargette, 1987) dans les trois secteurs de production visités. Cette échelle comprend cinq degrés ou notes variant de 0 à 4 . Ainsi, ont été definies la note $0=$ pas de symptôme ; $\mathbf{1}$ = légères mosaïques sans déformation et recouvrant moins de $20 \%$ de la surface foliaire; $\mathbf{2}$ = mosaïques et chloroses recouvrant environ $50 \%$ de la surface foliaire avec parfois déformation de la feuille ; $\mathbf{3}$ = mosaïques couvrant la majeure partie de la feuille accompagnée de nécroses, d'une déformation du limbe foliaire ; $\mathbf{4}$ = stade terminal caractérisé par la mort du cigare.

\subsection{Test sérologique des échantillons foliaires}

Le test DAS-ELISA a été utilisé pour confirmer la présence du virus dans les échantillons de feuilles de bananier. Un kit DAS-ELISA (DSMZ, Deutsche Sammlung Von Mikroorganismen und Zellkulturen) contenant des anticorps polyclonaux antiCMV (CMV DAS-ELISA/AS 0475/lgG (Immunoglobuline $\mathrm{G}$ ) et leurs conjugués (CMV DASELISA/AS 0475/lgG-AP (Alkaline Phosphatase) a été utilisé.

Une quantité de $500 \mathrm{mg}$ de feuilles a été broyée dans l'azote liquide, puis récupérée dans $10 \mathrm{ml}$ de tampon PBS-Tween, suivie d'une centrifugation à $5000 \mathrm{rpm}$ pendant $5 \mathrm{~min}$. Le test DAS-ELISA selon Clark et Adams (1977) a utilisé l'anticorps polyclonal (IgGs anti-CMV) et le conjugué marqué à la Phosphatase Alcaline (anti-CMV-PA) (DSMZ). La lecture de la Densité Optique (D.O ou absorbance à $405 \mathrm{~nm}$ ) a été effectuée à l'aide du lecteur de plaque ELISA Multiskan EX Labsystems 10.3.1999, 1 heure après incubation de la plaque ELISA à l'obscurité. Les échantillons pour lesquels la D.O était supérieure au seuil de positivité (3x moyenne DO des témoins non infectés) ont été considérés comme positifs. Sur la base du titre viral, différents niveaux d'infection ont été définis par rapport au seuil de positivité (SP). Ainsi, (+) pour DO $\in$ [SP ; 2SPI ; (++) pour DO $\in$ [2SP ; 3SP $;(+++)$ pour $\mathrm{DO} \geq 3 \mathrm{SP}$.

$(+),(++)$ et $(+++)$ correspondent respectivement à peu infecté, moyennement infecté et très infectés. (-) correspond à l'échantillon non infecté. 


\subsection{Distribution du CMV}

L'étude de la distribution du CMV a été réalisée sur la base des symptômes de la maladie observés dans les plantations. Elle a consisté à une description des types de foyers et leur emplacement par rapport à la parcelle. Des gradients d'infection ont été recherchés en notant l'importance des infections de la bordure au centre de la parcelle., Cela a permis d'évaluer l'importance relative des disséminations primaires et secondaires en relation avec l'abondance d'hôtes sauvages du CMV et de l'emplacement des parcelles infectées. Pour ce faire, des parcelles élémentaires d'observation de $30 \mathrm{~m} \times 5$ $\mathrm{m}$, en bordure des carrés ont été définies. Les carrés ont été ensuite parcourus de la bordure vers le centre.

\subsection{Incidence du CMV dans les pépinières et dans les plantations}

Le tri sanitaire des lots de plants (ensemble de plants issus du même méristème mère) effectué par les contrôleurs phytosanitaires a permis d'évaluer l'incidence du CMV dans les pépinières. Un lot est considéré infecté si au moins 1 plant de ce lot est infecté. L'incidence du CMV est le rapport du nombre de plants infectés sur le nombre total de plants du lot. De 2001 à 2005 un total de 747 lots a été examiné par les contrôleurs phytosanitaires. Ces lots ont été au cours de cette étude classés en trois différentes catégories en fonction du Taux d'Infection ( $\mathrm{TI})$ : Catégorie I $(\mathrm{TI}<5 \%)$, catégorie II $(5 \% \leq \mathrm{Tl} \leq 10 \%)$ catégorie III ( $\mathrm{Tl}>10 \%)$. La sélection des plantules pour le planting est effectuée après 45 jours de séjour en pépinière. L'évaluation de l'incidence du CMV dans les carrés au stade 3 MAP repose sur les données des contrôles phytosanitaires des secteurs Niéky et Banacomoé effectués en 2006. Ces contrôles ont été effectués régulièrement chaque 15 jours depuis le planting jusqu'à trois mois après le planting. L'incidence a été calculée à partir de la formule suivante :

$$
I=\frac{\sum_{t=1}^{6} P t}{N} \times 100
$$

Où, $P t=$ nombre de pieds malades à la période de contrôle ;

$N=$ Nombre total de plant du carré ;

$I=$ incidence.

\subsection{Cinétique de propagation de la maladie en plantation}

La cinétique de propagation du CMV a été appréciée par le suivi du nombre d'individus nouvellement infectés, par unité de temps (15 jours). Elle a été évaluée à partir des données de contrôles phytosanitaires de huit carrés choisis de façon aléatoire dans les plantations Agbo 2, Agbo 3 et Agbo 4 du secteur de Niéky durant la campagne 2006. Les pieds qui présentaient des symptômes caractéristiques du CMV ont été dénombrés et éliminés au cours de ces contrôles.

\subsection{Etude de l'incidence du CMV sur deux cycles successifs de cultures}

La technique culturale du bananier pratiquée dans ces secteurs de production, intègre une jachère d'un an ou deux, après un cycle de culture. Les données phytosanitaires des carrés 1, 2, 3, 4 et 5 de la plantation Comoé 3 du secteur Banacomoé, disponibles pour deux cycles successifs de culture, ont servi à cette étude. Les niveaux d'infection des carrés après ces deux cycles culturaux ont été comparés.

\section{Résultats}

\subsection{Sévérité des symptômes en pépinière et en plantation}

En pépinière, les symptômes observés ont consisté en de simples chloroses avec quelques plants présentant des symptômes d'intensité 3 à 4. Différents niveaux d'intensités de symptômes du CMV ont été observés dans les plantations sur des plants au stade 3 MAP (Fig. 1). Il a été observé pour un même pied, des symptômes d'intensité très variable (échelle 1 à 4) sur deux feuilles de rangs successifs. Les symptômes bien distincts sur les feuilles ont permis de classer les niveaux d'infection des plants suivant l'échelle de Cours. Les symptômes observés sur l'ensemble des 3 secteurs ont été ensuite rangés dans 3 intervalles de classes que sont [1;2[, [2;3[, [3;4[ et les proportions de plants atteints par intervalles de classe de symptômes ont été récapitulées (Tableau 1). De façon globale, les symptômes sévères de la maladie (classe [3; 4[), entraînant le plus souvent, une pourriture du cœur, sont prédominants $(61,25$ à $75 \%)$ sur l'ensemble des 
trois secteurs visités avec un taux de prévalence moyen de $66,25 \%$. Le taux le plus élévé (75\%) pour cette classe de sévérité, a été enregistré dans le secteur Banacomoé. La classe [2 ; 3 [ est présente avec une prévalence moyenne de $19,58 \%$. Les secteurs Niéky et Grand Fleuve ont enregistré un taux de prévalence $22,5 \%$ contre $13,75 \%$ à Banacomoé pour cette classe. La classe de symptômes la moins rencontrée dans les plantations visitées est la classe $[1 ; 2$
[ avec un taux moyen de $14,16 \%$. Les secteurs Niéky et Grand Fleuve ont enregistré les plus fort taux de prévalence pour cette classe avec $16,25 \%$ et $15 \%$ respectivement (Tableau 1 ). Au stade 8 MAP (stade de la floraison), la plupart des symptômes observés consistaient en des plages chlorotiques sur la hampe et le nanisme des doigts. Ces symptômes ont été particulièrement observés au secteur Niéky et au secteur Grand-fleuve.
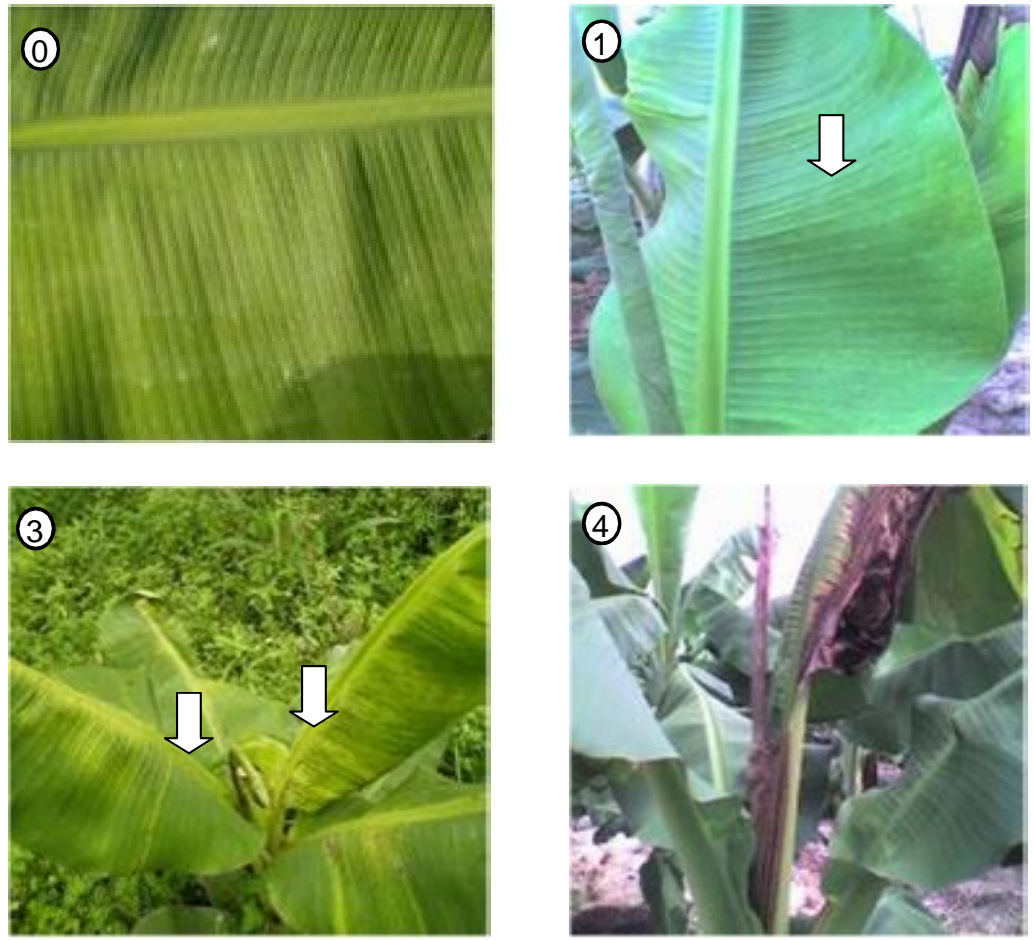

Figure 1 : Symptômes du CMV sur feuilles de bananiers dans des plantations industrielles de Niéky, grand-fleuve et Banacomoé.

$\mathbf{0}$ : pas de symptôme ; $\mathbf{1}$ : légères mosaïques sans déformation et recouvrant moins de $20 \%$ de la surface foliaire ; 3 : mosaïques couvrant la majeure partie de la feuille ; accompagnée de nécroses, d'une déformation du limbe foliaire ; 4 : stade terminal caractérisé par la mort du cigare.

Tableau 1 : Importance des classes de severité du CMV dans les secteurs de production de bananes.

\begin{tabular}{|c|c|c|c|c|}
\hline \multicolumn{2}{|c|}{ Classe de symptôme } & {$[1 ; 2[$} & {$[2 ; 3[$} & {$[3 ; 4[$} \\
\hline \multirow{4}{*}{ 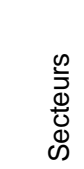 } & Niéky & $16,25 \%$ & $22,5 \%$ & $61,25 \%$ \\
\hline & Banacomoé & $11,25 \%$ & $13,75 \%$ & $75 \%$ \\
\hline & Grand Fleuve & $15 \%$ & $22,5 \%$ & $62,5 \%$ \\
\hline & Moyennes & $14,16 \%$ & $19,58 \%$ & $66,25 \%$ \\
\hline
\end{tabular}




\subsection{Diagnostic sérologique du CMV par le test ELISA}

Sur les 52 échantillons de feuilles présentant des symptômes de virose, les tests ELISA ont permis de confirmer l'infection par le CMV dans 49 échantillons (soit un taux de détection de $94 \%$ ) (Tableau 2). Sur les 7 échantillons supposés sains, prélevés au hasard dans le germoplasme de bananiers, un seul a été positif au test ELISA (soit une incidence de $14 \%$ ). En fonction du titre viral des échantillons virosés (50 échantillons testés positifs), 38 (76\%) se sont révélés très infectés, 8 (16 \%) considérés comme moyennement infectés et 4 ( $8 \%$ ) échantillons peu infectés (Tableau 2).

Tableau 2 : Résultats du test DAS-ELISA réalisé sur les échantillons de feuilles

\begin{tabular}{cccccc}
\hline $\begin{array}{c}\text { Code } \\
\text { échantillon }\end{array}$ & Statut/CMV & $\begin{array}{c}\text { Code } \\
\text { échantillon }\end{array}$ & Statut/CMV & $\begin{array}{c}\text { Code } \\
\text { échantillon }\end{array}$ & Statut/CMV \\
\hline B1 & +++ & $\mathrm{B}^{*}$ & - & $\mathrm{T} 15$ & +++ \\
B2 & +++ & ${\mathrm{B} 22^{*}}^{*}$ & - & $\mathrm{T} 16$ & ++ \\
B3 & + & ${\mathrm{B} 23^{*}}^{*}$ & - & $\mathrm{T} 17$ & ++ \\
B4 & +++ & ${\mathrm{B} 24^{*}}^{*}$ & - & $\mathrm{T} 18$ & +++ \\
B5 & +++ & ${\mathrm{B} 25^{*}}^{*}$ & - & $\mathrm{T} 19$ & +++ \\
B6 & +++ & $\mathrm{T} 1$ & ++ & $\mathrm{N} 1$ & +++ \\
B7 & +++ & $\mathrm{T} 2$ & +++ & $\mathrm{N} 2$ & +++ \\
B8 & ++ & $\mathrm{T} 3$ & +++ & $\mathrm{N} 3$ & +++ \\
B9 & + & $\mathrm{T} 4$ & +++ & $\mathrm{N} 5$ & +++ \\
B10 & - & $\mathrm{T} 5$ & +++ & $\mathrm{N} 7$ & ++ \\
B11 & +++ & $\mathrm{T} 6$ & +++ & $\mathrm{N} 8$ & +++ \\
B12 & ++ & $\mathrm{T} 7$ & +++ & $\mathrm{N} 10$ & +++ \\
B13 & +++ & $\mathrm{T} 8$ & +++ & $\mathrm{N} 11$ & +++ \\
B14 & - & $\mathrm{T} 9$ & +++ & $\mathrm{N} 12$ & +++ \\
B15 & + & $\mathrm{T} 10$ & +++ & $\mathrm{N} 17$ & - \\
B16 & +++ & $\mathrm{T} 11$ & ++ & $\mathrm{N} 18$ & ++ \\
B17 & +++ & $\mathrm{T} 12$ & +++ & $\mathrm{N} 19$ & +++ \\
B18 & +++ & $\mathrm{T} 13$ & +++ & $\mathrm{N} 20$ & +++ \\
B19* & + & $\mathrm{T} 14$ & +++ & $\mathrm{N} 21$ & +++ \\
B20* & - & & & $\mathrm{N} 22$ & +++ \\
\hline
\end{tabular}

Echantillons symptomatiques : 52 Positifs : 49 Négatifs : 03

Echantillons apparemment sains : 07 Positifs : 01 Négatifs : 06

*: échantillons de feuilles de la collection de génotypes (apparemment sains)

-: non infecté (9) ;peu infecté (4) ; moyennement infecté (8); très infecté (38)

$S P$ : seuil de positivité, $S P<D O \leq 2 S P$ : matériel peu infecté $(+) ; 2 S P<D O \leq 3 S P$ : matériel moyennement infecté(++) ;

$D O>3 S P$ : très infecté(+++)

\subsection{Distribution du CMV à l'échelle des plantations}

La distribution de la chlorose infectieuse du bananier sur l'ensemble des parcelles visitées, montre que l'infection n'était pas repartie de façon uniforme dans les parcelles. Elle met en évidence plusieurs zones endémiques avec différents types de foyers d'infection. Ces foyers observés dans les parcelles ont été en général, de types marginaux. La Figure 2 présente les proportions des différents types de foyers d'infection sur l'ensemble des secteurs, trois mois après le planting. L'incidence de la maladie dans $75 \%$ des cas, est apparue plus élevée sur les bordures des carrés et plus précisément le long des drains collecteurs. Les foyers d'infection de type généralisé ont été observés dans moins de $17 \%$ des cas sur l'ensemble des secteurs. Dans le secteur Grand-fleuve, les plantations sont marquées par une prédominance des foyers de types isolés (95\%). Dans les secteurs Niéky et Banacomoé, les foyers de type marginaux sont dominants $(70 \%)$. 


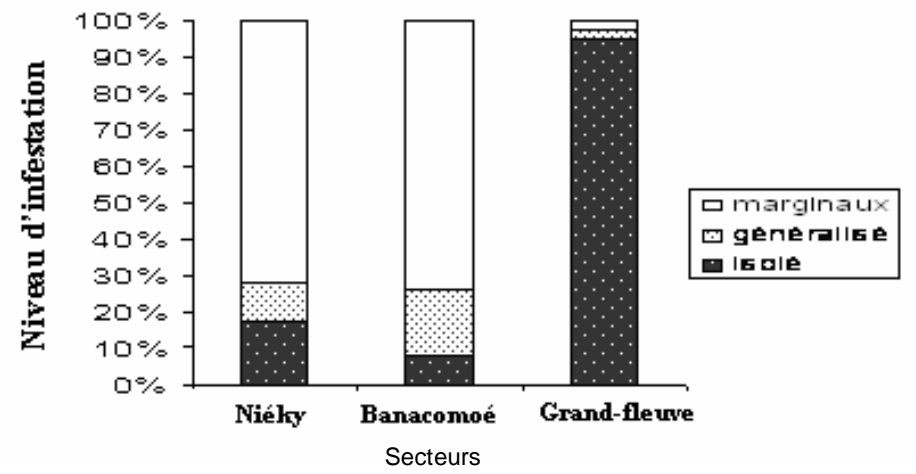

Figure 2 : Types et importance des foyers d'infection dans les plantations.

Niéky, Banacomoé et Grand-fleuve sont des secteurs de plantations industrielles

\subsection{Incidence du CMV dans les pépinières et dans les plantations}

\subsubsection{Dans les pépinières}

Les contrôles phytosanitaires dans les pépinières de Banacomoé de 2001 à 2005 ont révélé que $58,26 \%$ des lots de plantules (ensemble de plants issus du même méristème mère) en pépinière étaient infectés par le CMV. Une classification faite à partir du taux d'infection des lots (figure 3) a montré que :

- $\quad 87,80 \%$ des lots virosés, présentaient un taux d'infection inférieur à $5 \%$;

- $7,35 \%$ des lots virosés ont eu un taux d'infection compris entre $5 \%$ et $10 \%$;

- $\quad 4,85 \%$ des lots virosés ont présenté un taux d'infection supérieure à $10 \%$.

3.4.2. Incidence dans les bananeraies au stade 3 MAP et au stade 3-8 MAP,

L'incidence de la chlorose infectieuse du bananier dans les différents carrés des secteurs de Niéky (Dabou), Banacomoé (Abengourou) et de Grand-fleuve (Tiassalé) a été évaluée séparément pour le stade 3 MAP et le stade 3-8 MAP. Dans le secteur de Banacomoé, l'incidence du CMV dans les carrés était comprise entre 2 et $23,7 \%$ (Fig. 4). Le taux moyen de plants infectés dans les carrés du secteur de Banacomoé a été estimé à $6,16 \%$. Le carré 5 de la plantation Comoé 3 , a été le plus infecté. Avec $23,7 \%$, ce carré a affiché plus de la moitié des nouveaux cas de viroses enregistrés sur l'ensemble des plantations du secteur Banacomoé au cours de l'année 2006. Au secteur Niéky, la moyenne d'incidence du CMV dans les carrés était comprise entre 0,06 et 10,28 $\%$ (Fig. 5). La moyenne de l'incidence du CMV au secteur Niéky a été de 2,66\%. L'incidence de la chlorose infectieuse du bananier au secteur Grand-fleuve, a été très faible voire nulle au stade 3 MAP avec des valeurs de $0,12 \%$ dans le bloc M'brimbo 7 et de $0,06 \%$ dans le bloc M'brimbo 6. De même, l'incidence du CMV dans les plantations de ce secteur était quasiment nulle dans les plantations au stade 3-8 MAP.

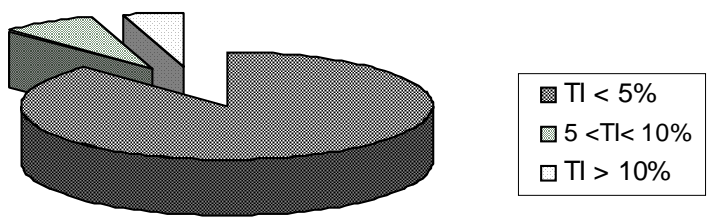

Figure 3 : Taux d'infection du CMV dans les lots de plantules en pépinières (2001-2005)

TI : taux d'infection 


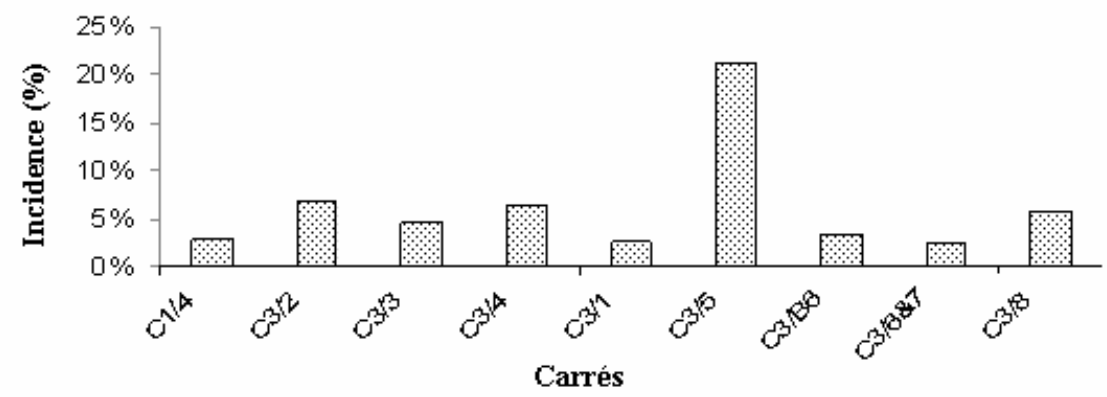

Figure 4: Incidence du CMV dans les carrés de moins de trois mois de Banacomoé en 2006

L'incidence du CMV a été calculée à partir des données des relevés phytosanitaires. Le nombre total de plants sur chacune des parcelles varie de 3960 à 12381 pour une superficie comprise entre 2,14 et 6,69 ha respectivement

C1/4 = Comoé 1 Carré $4 ; C 3 / 2=$ Comoé 3, Carré $2 ; C 3 / 3=$ Comoé 3, Carré $3 ; C 3 / 4=$ Comoé 3, Carré 4 ; C3/4 = Comoé 3, Carré 4 ; C3/5 = Comoé 3, Carré $5 ; C 3 / B 6=$ Comoé 3, Carré B6; C3/6 \& $7=$ Comoé 3, Carré 6 et $7 ;$ C3/8 = Comoé 3, Carré 8 ;

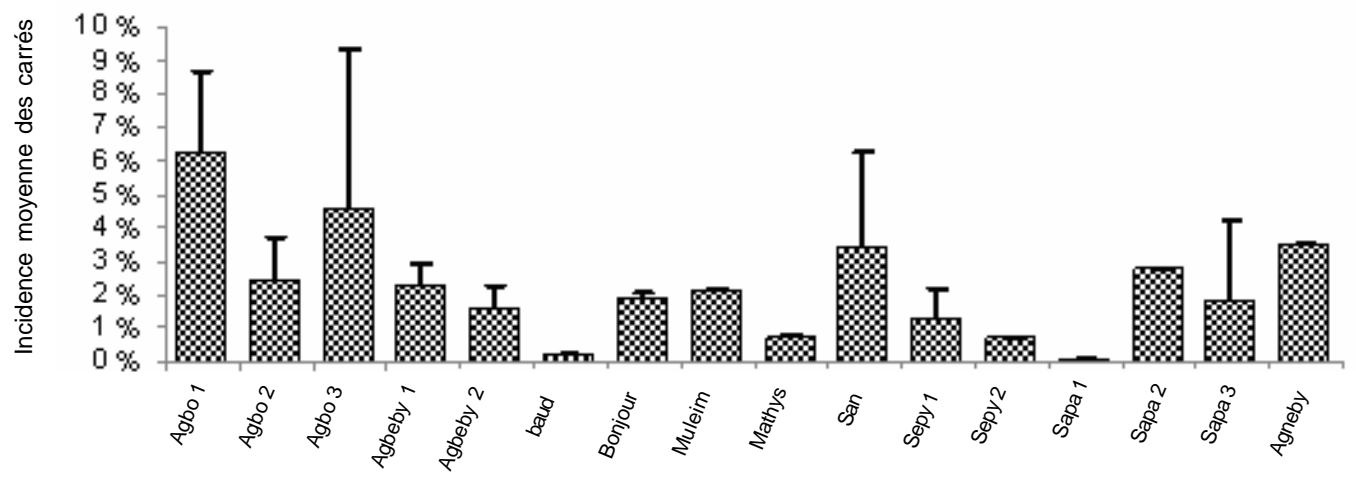

Figure 5 : Incidence du CMV dans les plantations de moins de trois mois de Niéky en 2006

\subsection{Cinétique d'évolution de la maladie en plantation}

Les courbes d'épidémies du CMV dans les huit carrés du secteur de Niéky, ont présenté en dépit des variations observées, une allure générale en forme de S. Elles présentent trois phases dont l'importance varie d'un carré à un autre. Une phase de faible croissance correspondant à une période d'incubation qui dure 15 à 45 jours, une phase exponentielle qui dure deux à quatre semaines et une phase stationnaire (saturation) de durée variable (Fig. 6). Aussi, les valeurs des pentes pour les différentes phases des courbes d'épidémie varient-elles d'un carré à l'autre. Les courbes des vitesses de dissémination de la maladie dans les carrés, sont très variables. Elles ont de façon générale une allure en cloche unimodale (carrés 4, 6, 11, 10, 14 et 41) ou bimodale (carrés 54, et 15) (Fig. 7). Les infections des plants sains sélectionnés en pépinière, ont débuté entre le $15^{\mathrm{e}}$ et $30^{\mathrm{e}}$ Jours Après Planting (JAP). Le nombre de plants infectés a rapidement augmenté à partir de $45 \mathrm{JAP}$ pour atteindre leur pic 60 JAP. A partir de $60 \mathrm{JAP}$, les taux des nouveaux cas (plants virosés) diminuent jusqu'à $75 \mathrm{JAP}$. (Fig. $7)$. Les vitesses de dissémination de la maladie ont été plus élevées dans les carrés 6 et 11 . 

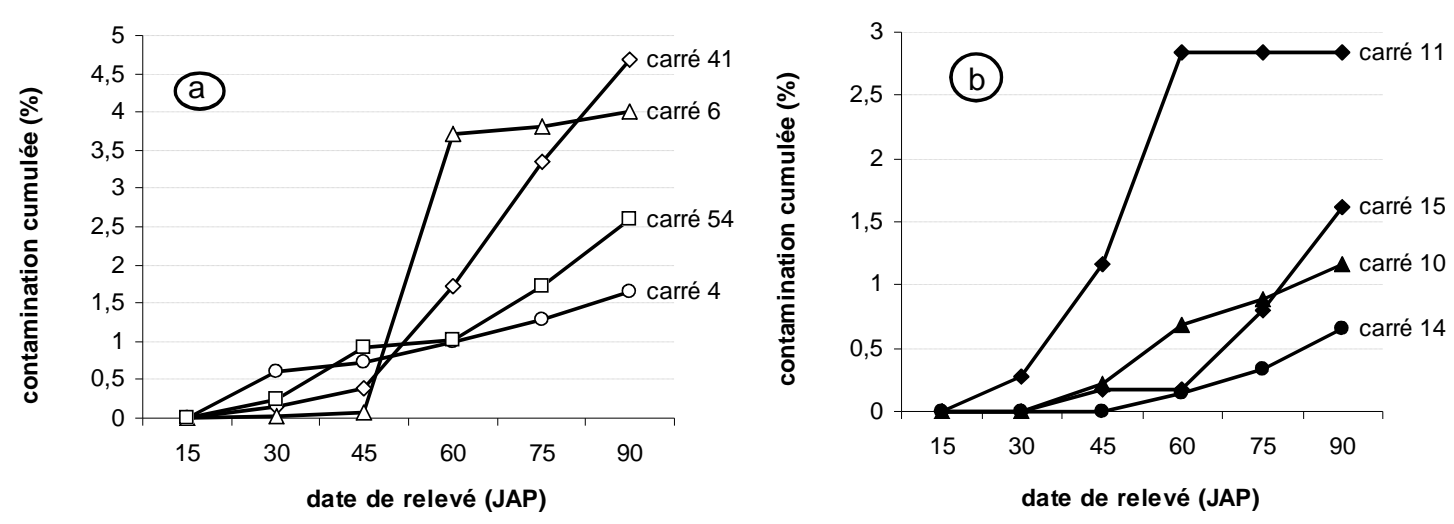

Figure 6 : Courbe de la progression de l'épidémie du CMV de huit carrés du secteur Niéky.

$J A P=$ jours après planting

a. progression de l'épidémie dans les carrés 4, 6, 41 et 54

b. progression de l'épidémie dans les carrés 10,11, 14 et 15
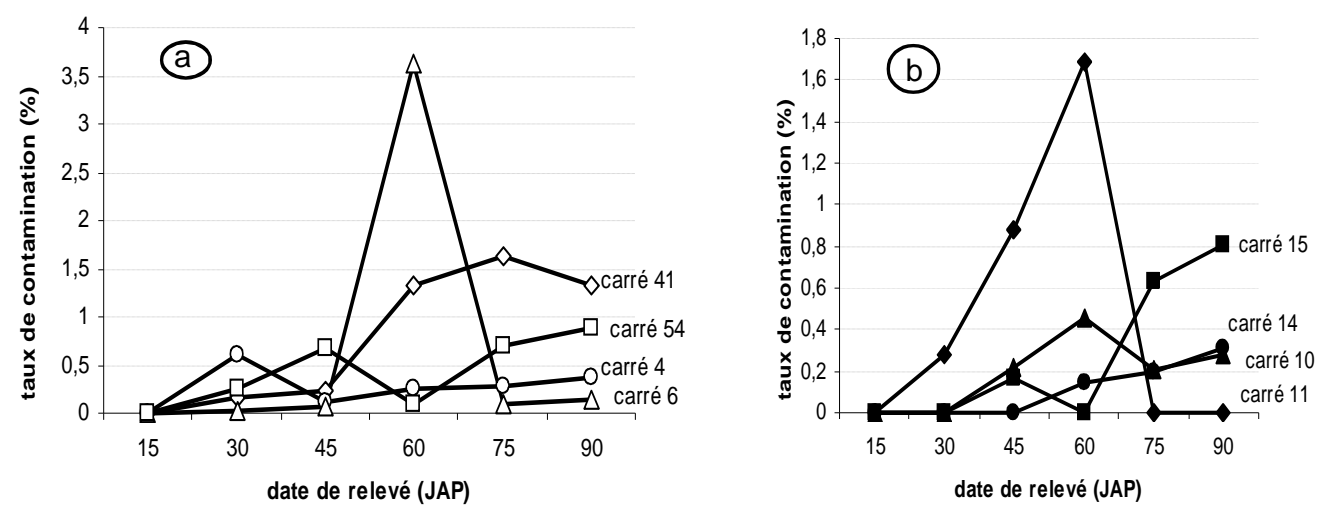

Figure 7 : Courbe de la cinétique de dissémination du CMV dans huit carrés du secteur Niéky.

$J A P=$ jours après planting

a. Cinétique de dissémination du CMV dans les carrés 4, 6, 41 et 54

b. Cinétique de dissémination du CMV dans les carrés 10,11, 14 et 15

\subsection{Comparaison de l'incidence du CMV de deux cycles successifs de cultures}

Les incidences moyennes du CMV entre deux cycles successifs de cultures (replantations) dans les carrés $1,2,3,4$ et 5 du bloc 3 du secteur de Banacomoé ont été étudiées. Au premier cycle de culture, les moyennes d'incidence de 0,82 à $2,95 \%$ ont été enregistrées tandis que ces taux sont passés de $2,10 \%$ à $17,90 \%$ au second cycle de plantation (Fig. 8). Le taux moyen de l'incidence du CMV au second cycle a été $7,23 \pm 5,42 \%$ alors qu'il n'était que de seulement
$1,70 \pm 0,75 \%$ au premier cycle. L'écart de l'incidence de la maladie entre deux replantations successives dans les carrés concernés a varié de 0,85 à 16,1. En général, il a été observé une tendance à la croissance très marquée des niveaux d'incidence du CMV dans les carrés à la seconde replantation. En effet, les incidences du CMV au second cycle dans les carrés 2, 3, 4 et 5 ont connu une évolution positive avec des écarts respectifs de 5,$38 ; 3,05$; 3,95 et 16,1 . Quant au carré 1, il a enregistré une faible diminution de l'incidence du CMV avec un écart de 0,85\% (Fig. 8). 


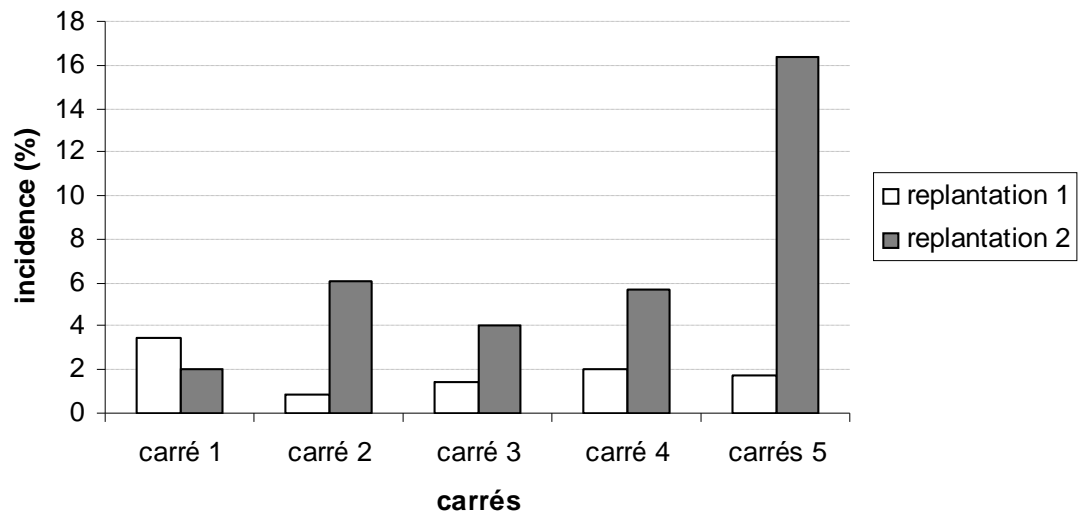

Figure 8 : Comparaison de l'incidence du CMV de deux cycles de plantations successifs. Les valeurs utilisées sont des taux moyens calculés.

\section{Discussion}

La mosaïque du bananier causée par Cucumber mosaic virus (CMV) est présente dans tous les secteurs de production de banane couverts par notre étude à savoir Niéky (Dabou), Banacomoé (Abengourou) et de Grand-fleuve (Tiassalé). Le nombre de parcelles visitées a été fonction de l'importance des surfaces emblavées dans ces différents secteurs de production. Les trois secteurs visités ont présenté des incidences variables avec une prédominance des symptômes d'intensité 4 (stade terminal) dans les plantations de moins de 3 mois. Cette observation pourrait être liée à la présence de souches virales de CMV induisant des symptômes sévères. Ces types de symptômes sont généralement causés par les pathotypes du CMV appartenant au sous-groupe I (Fakhfakh et al., 2002). De plus les travaux de séquençage du génome de 16 isolats du CMV de Côte d'Ivoire collectés dans la zone d'étude ont montré une homologie de 99,2 \% en nucléotide avec les isolats de CMV appartenant au sousgroupe IA (Kouassi, travaux non publiés). Cette forte présomption de la présence de ce pathotype differe des résultats de Lockhart (2000) qui affirmait le contraire en Afrique. Des tests d'inoculations des isolats, en particulier ceux qui provoquent la pourriture du pseudo-tronc et du cœur du bananier et le nanisme des doigts sur des variétés sensibles sont nécessaires pour confirmer nos observations. Aussi la prise en compte des stades phénologiques dans cette étude permettra-t-elle, d'évaluer la corrélation entre l'agressivité des isolats et l'âge de la plante.
Le test de diagnostic sérologique de type ELISA utilisé est facile à mettre en œuvre et peut être préconisé pour l'indexage en routine des rejets de bananier utilisés en culture in vitro. Des échantillons présentant des symptômes de virose, ont été testés négatifs. A l'opposé, ce test a mis en évidence la présence de virus dans des échantillons de feuilles apparemment sains. Ces résultats indiquent que le diagnostic des maladies virales uniquement à partir des symptômes exprimés n'est pas toujours fiable. En effet, les symptômes causés par un virus particulier peuvent se confondre à un autre (Astier et al., 2001). Dans notre cas les symptômes du CMV peuvent parfois se confondre à ceux du BSV. La confirmation des observations visuelles par des tests fiables comme les tests ELISA et les tests moléculaires est nécessaire. Les symptômes du CMV vraisemblablement observés sur les trois plants ayant réagi négativement à l'anticorps dirigé contre le CMV peut être un cas de confusion avec le BSV. Le cas de l'échantillon apparemment sain testé positif, pourrait s'expliquer par la sensibilité des tests utilisés pouvant détecter de façon précoce la présence du pathogène avant l'apparition des symptômes.

La répartition ou la distribution spatiale de la maladie dans les plantations prospectées dépend pour une large part, des techniques culturales comme le type d'irrigation qui influence considérablement la propagation de la maladie dans les carrés. En effet, à Niéky et à Banacomoé où l'irrigation se fait par aspersion, l'enherbement est plus abondant qu'à Grand-fleuve où l'irrigation est de type goutte à goutte. Dans le premier cas, la 
prolifération des adventices et les populations de pucerons qu'ils pourraient héberger sont des sources potentielles d'infection contribuant à la dissémination de la virose dans les bananeraies. En outre, la présence de plusieurs plantations villageoises ne bénéficiant pas de surveillance sanitaire, constitue des sources majeures d'inoculum.

L'évaluation de l'incidence dans la pépinière de Banacomoé à partir des symptômes a révélé que plus de $50 \%$ des lots de plants en pépinière (au stade juvénile) étaient infectés. Les taux d'infection calculés à partir des données de relevés de plants virosés (comptage des plants symptomatiques) ont été généralement inférieurs à $5 \%$, cependant des taux d'infection supérieurs à $10 \%$ ont été parfois enregistrés $(4,83 \%$ des cas) en dépit des mesures de protection dont cette pépinière fait l'objet. On peut donc penser que les infections sont probablement issues du matériel végétal (rejets) ayant servi à l'obtention des vitroplants au laboratoire. Dans ce cas, les lots présentant un taux d'infection supérieur à $10 \%$, devraient faire l'objet d'une étude dans le but de tester les pieds «mères» à partir desquels les méristèmes ont été prélevés pour s'assurer de leur état sanitaire initial.

L'incidence du CMV calculée à partir des relevés phytosanitaires dans le secteur Grand-fleuve, a été très faible voire nulle dans les plantations au stade 3 MAP avec des valeurs de $0,12 \%$ dans le bloc M'brimbo 7 et de $0,06 \%$ dans le bloc M'brimbo 6. Les faibles incidences du CMV enregistrées dans ce secteur Grand-fleuve, sont en grande partie, liées à la maîtrise de l'enherbement dans les parcelles. Le secteur Niéky a enregistré une moyenne d'incidence du CMV de 2,66 \% au cours de nos études. Cependant, les valeurs de l'incidence pour certains carrés pouvaient parfois atteindre $40 \%$ au cours des années antérieures. Dans le secteur de Banacomoé, l'incidence du CMV dans les carrés a varié entre 2 et $23,7 \%$ environ. La grande variabilité de l'incidence du CMV enregistré d'une plantation à une autre et parfois à l'intérieur d'une même plantation pourrait s'expliquer par un effet environnement significatif. En effet, l'emplacement des carrés par rapport aux drains collecteurs des eaux d'irrigation favorisant le développement des réservoirs sauvages du CMV et l'abondance des populations d'insectes vecteurs constituent une source de variabilité non négligeable. Pour les carrés à proximité des drains collecteurs, les risques de contamination sont plus élevés.

La cinétique de contamination a montré une fluctuation des infections au cours du temps; ce qui correspond à une variation de la pression de l'inoculum dans les carrés. Le temps d'apparition des premiers symptômes du CMV s'est situé entre 15 et $30 \mathrm{JAP}$, ce qui correspond à la durée d'incubation du CMV qui se situe entre 15 et 30 jours suivant les spéculations (Jacquemond \& Lot, 1982). L'augmentation des taux d'infections jusqu'a 45 JAP peut résulter des contaminations mécaniques dues à l'usage commun des outils de taille tels que les coupe-feuilles et les gouges utilisés par les exploitants. Elle peut aussi être associée au stade phénologique des plants favorables aux contaminations. En effet, il a été prouvé qu'une plante en forte croissance est plus sensible à l'infection (Fargette, 1987 ; Hwand \& $\mathrm{Su}, 1998)$. Les phases de décroissance des infections enregistrées peuvent s'expliquer non seulement par la lutte mécanique consistant à une élimination systématique des plants infectés, mais aussi par un renforcement des mécanismes ou facteurs de résistance liés à l'âge de la plante. L'absence de la virose dans les plantations de plus de trois mois d'âge au cours de cette étude, semble confirmer la seconde hypothèse. Le modèle mathématique pour les contaminations à intérêts simples $(\mathrm{dx} / \mathrm{d} t$ $=r x(1-x))$ défini par Fargette (1987) lié à la progression de l'épidémie du CMV ne s'applique que dans les carrés 6 et 11. Dans les autres carrés, la phase logistique de la courbe se prolonge et ne permet pas d'apprécier véritablement la phase terminale de la courbe de contaminations.

L'étude de la progression de l'incidence entre deux cycles de plantations successives, montre une tendance générale à l'augmentation au second cycle. Ce phénomène pourrait s'expliquer entre autre par l'utilisation des plantes potentiellement hôtes du virus et la prolifération des insectes vecteurs dans les jachères.

\section{Conclusion}

Cette étude a permis de faire l'état des lieux de la mosaïque du bananier causée par le CMV dans les secteurs de productions industrielles de banane au Sud-Est de la Côte d'Ivoire. Les travaux effectués ont montré une fluctuation de la 
maladie dans l'espace et dans le temps. Les incidences dans les carrés oscillent entre $5 \%$ et $25 \%$ dans les plantations de 3 mois tandis qu'elles ont été quasiment nulles dans les plantations de plus de trois mois. Il a été observé une tendance à l'augmentation de l'incidence du CMV d'un cycle de plantation à un autre. En pépinière plus de $50 \%$ des lots de plants de bananier ont été trouvés infectés avec des taux d'infection parfois supérieurs à $10 \%$. L'identification et l'indexage par test sérologique des plantes mères desquelles, sont issus ces lots, s'avèrent donc nécessaire pour éviter la mise en culture d'explants déjà virosés. L'utilisation du test DAS-ELISA pourrait permettre cet indexage de façon spécifique en l'absence de symptômes visibles sur les feuilles. La cinétique de l'évolution de la maladie a montré que les premiers symptômes de la maladie sont observés entre 15 et $30 \mathrm{JAP}$ et les pics sont atteints autour de $60 \mathrm{JAP}$. En outre, l'étude de la répartition de la maladie à l'échelle de la parcelle (carré) a mis en évidence plusieurs foyers marginaux généra-lement situés le long des drains collecteurs des eaux d'irrigation. Les mauvaises herbes qui y poussent sont des réservoirs potentiels non seulement du virus mais aussi des insectes vecteurs de cette virose. Ces mauvaises herbes pourraient par conséquent constituer des facteurs non négligeables de dissémination de la mosaïque du bananier causée par Cucumber mosaïc virus.

\section{Remerciements}

Les auteurs remercient Dr Kobenan, chercheur du programme Ananas-Banane du CNRA pour ses critiques et suggestions pour l'amélioration de cette publication.

\section{Références citées}

Abadie C., Bakry F., Carlier J., Caruana M.L., Cote F., Ganry J., Lescot T., Marie P. \& Sarah J.L., 2004. Banana for Ever. Revue FruiTrop 99 : 4-10.

Astier S., Albouy J., Maury Y. and. Lecoq H, 2001. Principes de virologie végétale (Génomes, Pouvoir Pathogène, Ecologie des Virus) Paris, France : INRA. 444 pp.
Caruana M.L., 2007. Des outils de détection polyvalents pour les virus du bananier. Lettre d'information n²1 CIRAD, France, août 2007.

Clark M.F. \& Adams A.N., 1977. Characteristics of the microplate method of enzyme-linked immunosorbent assay for the detection of plant viruses. J. Gen. Virol. 34 : 475-483.

Cours G., 1950. Le manioc à Madagascar. Thèse de doctorat, Université de Paris, France. 417 pp.

Diekmann D.R. \& Putter C.A.J., 1996. Technical Guidelines for the Safe Movement of Germplasm, Musa, 2nd Ed., FAO/IPGRI, (15), Food and Agriculture Organization of the United Nations Rome/International Plant Genetic Resources Institute, Rome, 28 pp.

Fargette D., 1987. Epidémiologie de la Mosaïque Africaine du Manioc en Côte d'Ivoire ; Collection Etude et Thèse, Ed., ORSTOM, 203 pp.

Fakhfakh H., Gorsane H., Acheche H., Elleuch A., Djilani F., Feki-Hassen I., Strosse H., Domergue R., Panis B. \& Escalant J., 2003. Suspensions cellulaires embryogènes de bananiers et bananiers plantain; Guides techniques INIBAP 8, 5 pp.

FAOSTAT |, 2009. http://faostat.fao.org/site/567/ DesktopDefault.aspx?PagelD $=567$

Fauquet C. \& Thouvenel J.C., 1987. Maladies virales des plantes en Côte d'Ivoire/Plant viral Diseases in the Ivory Coast. Ed., ORSTOMParis 2e éd. (1987) 243 pp.

Hwang S. \& Su H., 1998. Production of virus-free banana plantlets in Taiwan, (page consultée le 04 février 2007), <http//www. agnet .org>, 26 p.

Jacquemond, M. \& Lot, H., 1982. L'ARN satellite du virus de la mosaïque du concombre III. La propriété de survie in vivo. Agronomie 2: 533-538.

Jones D.R., 1994. Risks Involved in the transfer of banana and plantain germplasm $I n$ : The Improvement and Testing of Musa: a Global Partnership. INIBAP, Montpellier, France. pp. 85-98.

Kouassi K.S., 2001. Les secteurs banane (AAA) et banane plantain $(A A B)$ en Côte d'lvoire : analyse bibliographique et prospective. Ed., CNRA. 35 pp. 
Lassoudière A., 1974. La Mosaïque dite à "tirets" du bananier 'poyo' en Côte d'Ivoire. Fruits 29, 349-357.

Lockhart B.E.L., 2000. Virus diseases of Musa in Africa: Epidemiology, detection and control $I n$ : banana and plantain for Africa. pp. 355-356.

Lockhart B.E.L., 2002. Management of viral diseases of banana. In: Acorbat- fitoprotección Memorias XV reuniòn Cartagena, Combias pp 217-221.

Rapilly F., 1991. L'épidémiologie en pathologie végétale, Ed., INRA, 317 pp.

Thresh J.M. \& Fargette D., 2001. The epidemiology of African plant viruses: basic principles and concepts In Plant virology in sub-saharan Africa, pp. $61-111$ 